\title{
Encefalitis amebiana granulomatosa por Balamuthia mandrillaris: una enfermedad fatal reconocida cada vez más frecuentemente en América Latina
}

\author{
Carlos Seas R. y Francisco Bravo P.
}

\author{
Amebic granulomatosis encephalitis due to Balamuthia mandrillaris: \\ a fatal disease increasingly recognized in Latin America
}

P ocas enfermedades infecciosas del sistema nervioso central (SNC) portan tan pobre pronóstico y causan en el médico tal sensación de pesimismo e impotencia como las producidas por las amebas de vida libre. Descubiertas como causa de infección del SNC en humanos desde 1965, las amebas de vida libre están asociadas a dos entidades muy diferentes en sus manifestaciones clínicas y hallazgos anátomo-patológicos: meningitis amebiana primaria (MAP) causada por Naegleria fowleri, y encefalitis amebiana granulomatosa (EAG) causada por Acanthamoeba sp, Balamuthia mandrillaris, o más recientemente por Sappinia diploidea. Mientras que MAP sigue un curso muy agudo caracterizado por meningitis hemorrágica, EAG sigue un curso más sub-agudo o crónico, en que el compromiso de la piel y lesiones metastásicas cerebrales son las características más saltantes ${ }^{1}$. Ambas entidades clínicas portan un pésimo pronóstico, con significativa mortalidad, pese a haberse ensayado tratamientos con una variedad de antibacterianos, antiparasitarios y antifúngicos.

Las amebas de vida libre son protozoarios que tienen en común varias características: no están bien adaptados al parasitismo, pues terminan con la vida del hospedero que invaden, no dependen de un hospedero o vector para su transmisión, y no se transmiten de humano a humano. Viven en el suelo y en el agua, especialmente $N$. fowleri. Los agentes de EAG son adquiridos presumiblemente a través de la piel o por vía inhalatoria, mientras que la MAP es adquirida por invasión nasal con posterior diseminación por el bulbo olfatorio hacia las meninges. Enfermedad clínica por estas amebas ha sido descrita en hospederos inmunocomprometidos e inmunocompetentes. No constituyen un problema de salud pública, como sí lo son las enfermedades producidas por otros protozoarios como malaria, amebiasis intestinal o tripanosomiasis, aunque su verdadera prevalencia es desconocida. Balamuthia mandrillaris, inicialmente identificada como causa de encefalitis en mandriles, ha sido reconocida como patógeno en humanos desde $1993^{1}$. Desde esa fecha, más de 100 casos han sido reportados en la literatura médica, provenientes de Australia, los Estados Unidos de Norte América, Japón, República Checa, y Latinoamérica, incluyendo Argentina, Brasil, México, Perú y Venezuela ${ }^{2-5}$. La enfermedad es muy poco reconocida en Europa y en el Sur Este de Asia ${ }^{6}$. Oddó y colaboradores reportan en este número de la revista el primer caso de EAG por B. mandrillaris diagnosticado en Chile, contribuyendo así a la cada vez mayor predominancia de reportes de esta entidad provenientes de América Latina.

Después de la serie norteamericana de EAG por B. mandrillaris, con aproximadamente 50 casos reportados, la experiencia mexicana con 45 casos y la peruana son las más numerosas. Perú reporta casos desde 1999; a la fecha 45 casos de EAG han sido observados, 20 de estos casos fueron causados por $B$. mandrillaris ${ }^{2,7,8}$. De esta serie pueden destacarse varias contribuciones. La mayoría de los casos ocurrió en niños varones, aunque no se había reconocido la enfermedad en un lactante de 7 meses como uno de los casos publicados en este número9. La edad fluctuó entre 5 y 63 años, el 50\% de los casos tuvo menos de 15 años. Los pacientes procedían en su mayoría de zonas desérticas, cálidas, costeras y rurales de tres departamentos del Perú, con amplia exposición recreacional a fuentes de agua provenientes de lagunas o lagos de agua dulce. No se observó ninguna forma conocida de inmunosupresión en los pacientes. La presentación clínica siguió un patrón común, de tal modo que el diagnóstico fue sospechado casi siempre pre-morten. La enfermedad se inicia con una o varias lesiones cutáneas, preferentemente de localización centrofacial, sobre la nariz o en el pómulo. La lesión cutánea es una placa de color rojo oscuro o violáceo, poco dolorosa, de pocos milímetros de espesor por algunos centímetros
Universidad Peruana Cayetano Heredia. Lima, Perú. Instituto de Medicina Tropical Alexander von Humboldt. Hospital Nacional Cayetano Heredia. Lima, Perú. Departamento de Enfermedades Infecciosas, Tropicales y Dermatológicas.

Correspondencia a: Carlos Seas Ramos cseas@upch.edu.pe 


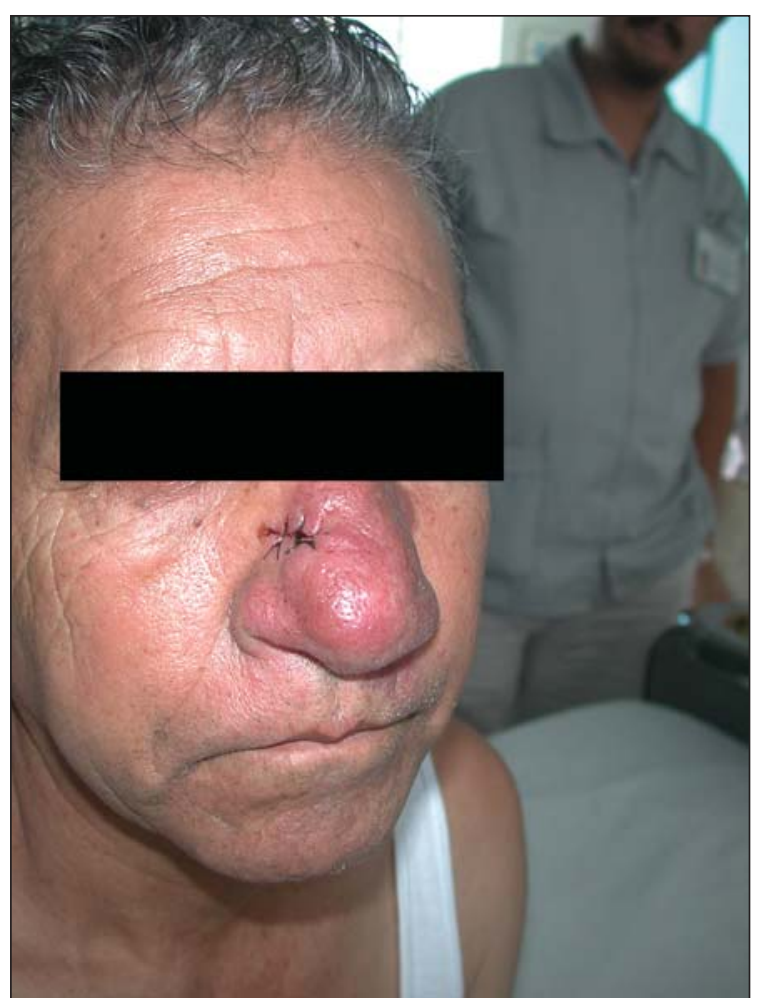

de ancho, rara vez se ulcera (Figura 1). A la palpación, la lesión tiene una consistencia indurada. El diagnóstico diferencial debe incluir lupus vulgaris, leishmaniasis, esporotricosis, rinoescleroma, granulomatosis de Wegener y cáncer. En países tropicales debiera incluirse en esta lista a las paracoccidiodomicosis y lepra. Semanas a meses después del inicio de la lesión cutánea, 5 a 8 meses en promedio, ocurren las manifestaciones del SNC, caracterizadas por signos focales deficitarios, hipertensión endocraneana, crisis convulsivas y compromiso progresivo del sensorio. El aspecto de las lesiones cerebrales a la TAC o RM es la de múltiples lesiones hipodensas rodeadas de un halo inflamatorio. La presentación con signos neurológicos, sin lesión cutánea, es la excepción más que la regla en la experiencia peruana, en contraposición con lo reportado por otros autores. Cuando esto ocurre, como en el caso en discusión, el diagnóstico fue hecho tardíamente. Otros diagnósticos fueron considerados antes que EAG en estos casos, incluyendo tuberculomas cerebrales, cisticercosis, metástasis cerebrales y toxoplasmosis. Una detallada descripción de los hallazgos patológicos cerebrales fue hecha por Recavarren-Arce y colaboradores $^{10}$. Destaca en su reporte el hallazgo de perivasculitis amebiana, que conduce a trombosis y microinfartos cerebrales, proceso que facilitaría la invasión ulterior del parénquima cerebral. A pesar de un diagnóstico excepcionalmente temprano, y a pesar de variados intentos terapéuticos, sólo 4 pacientes han sobrevivido en la serie peruana, lo que confirma el pésimo pronóstico que esta entidad tiene. Dos pacientes sobrevivieron luego de producirse compromiso neurológico, ambos casos fueron tratados con cursos largos de albendazol e itraconazol. La experiencia con anfotericina B, pentamidina, cotrimoxazol, metronidazol, rifampicina y fluconazol ha sido muy desalentadora. El caso reportado en este número tiene así algunas características inusuales para la experiencia peruana, como la edad y la presentación clínica sin lesión cutánea.

Varias contribuciones al mejor conocimiento de EAG por B. mandrillaris han ocurrido recientemente. En primer lugar, ha sido demostrada alteración de la barrera hemato-encefálica inducida por liberación de interleuquina $6^{11}$. Este hallazgo sugeriría que las amebas inducen una respuesta inflamatoria que altera la permeabilidad de la barrera hemato-encefálica, favoreciendo la diseminación de la infección. Con-

Figura 1. Lesión nasal característica causada por Balamuthia mandrillaris. gruente con este hallazgo son las anormalidades observadas en el LCR, consistentes en pleocitosis a predominio linfo-mononuclear con proteinorraquia. Este hallazgo también sugiere que antimicrobianos que se concentren en el SNC con la barrera hemato-encefálica alterada tendrían mejor eficacia clínica. En segundo lugar, el primer aislado de B. mandrillaris del LCR ha sido reportado recientemente en un paciente proveniente de Bolivia ${ }^{12}$. Sin embargo, el único aislado de $B$. mandrillaris en directa relación con un caso clínico se hizo de una maceta del jardín de la casa donde residía el niño afectado ${ }^{13}$. La identificación pre-morten de $B$. mandrillaris no es nada sencilla, descansa en el hallazgo histológico del trofozoito o quiste, confirmado por inmunofluorescencia. Es por ello que la tercera contribución consistente en un reporte de aplicación de una técnica de RPC a especímenes clínicos es relevante. La técnica es aplicable al LCR y tejidos, alcanzando alta sensibilidad y especificidad, abriendo la posibilidad de un diagnóstico más temprano ${ }^{14}$. Es interesante notar el predominio de origen latinoamericano en pacientes con EAG por B. mandrillaris. No existe una explicación razonable para este hallazgo, han sido planteadas hipótesis como mayor exposición o factores genéticos; esta asociación necesita ser mejor estudiada ${ }^{1}$. Finalmente, existen reportes de tres pacientes en Norteamérica que han sobrevivido a la invasión del SNC por esta ameba ${ }^{15,16}$. Todos ellos recibieron por tiempo prolongado una combinación de antimicrobianos que incluía pentamidina, fluocitosina, fluconazol, claritromicina y sulfadiazina. Se observaron múltiples reacciones adversas a esta combinación de antimicrobianos. Sumando estos casos a los 4 casos peruanos, tendríamos un total de 7 sobrevivientes reportados en la literatura médica. Sin embargo, no es posible recomendar un esquema de tratamiento único a partir de esos reportes.

El pesimismo actual sobre el pronóstico de pacientes con EAG podría cambiar si conociéramos mejor la prevalencia de la enfermedad y su modo de transmisión, si tuviéramos disponible métodos de diagnóstico rápido y conociéramos mejor la susceptibilidad del agente a los antimicrobianos. Mientras se genere más información sobre estos temas, es necesario tener un bajo umbral para considerar el diagnóstico de EAG en todo paciente con compromiso cutáneo sugestivo, y en pacientes con lesión neurológica compatible de causa no precisada. 


\section{Referencias}

1.- Schuster F L, Visvesvara G S. Free-living amoebae as opportunistic and nonopportunistic pathogens of humans and animals. Int J Parasitol 2004; 34: 1001-27.

2.- Bravo F, Sánchez M R. New and re-emerging cutaneous infectious diseases in Latin America and other geographic areas. Dermatol Clin 2003; 21: 655-68.

3.- Tarauto A L, Monges J, Acefe J C, Meli F, Paredes A, Martínez A J. Leptomyxid amoeba encephalitis: report of the first case in Argentina. Trans Royal Soc Trop Med Hyg 1991; 85: 77.

4.- Chimelli L, Hahn M D, Scaravelli F, Wallace S, Visvesvara G S. Granulomatous amoebic encephalitis due to leptomyxid amoebae: report of the first Brazilian case.

Trans Royal Soc Trop Med Hyg 1992; 86: 635-6.

5.- Riestra-Castañeda J M, Riestra-Castañeda R, González-Garrido A A, et al. Granulomatous amebic encephalitis due to Balamuthia mandrillaris (Leptomyxidae): report of four cases from Mexico. Am J Trop Med Hyg 1997; 56: 603-7.

6.- Intalapaporn $\mathrm{P}$, Suankratay $\mathrm{C}$, Shuangshoti S, Phantumchinda K, Keelawat S. Wilde H. Balamuthia mandrillaris meningoencephalitis: the first case in South East Asia. Am J Trop Med Hyg 2004; 70 : 666-9.

7.- Campos P, Cabrera J, Gotuzzo E, Guillén D. Neurological involvement in free living amebiasis. Rev Neurol 1999; 9: 316-8.

8.- Bravo F G, Cabrera J, Gotuzzo E. Cutaneous manifestations of infection by free living amebas, with special emphasis on Balamuthia mandrillaris. En: Tyring SK, Lupi O, Henage UR, eds. Tropical Dermatology. Philadelphia: Churchill Livingstone; 2005: 49-56.

9.- Cuevas M, Smoje G, Jofré L, Ledermann W, Noemí J, Berwart C, et al. Meningoencefalitis granulomatosa por Balamuthia mandrillaris: Reporte de un caso y revisión de la literatura. Rev Chil Infect 2006; 23: 234-9 (en este número).

10.- Recavarren-Arce S, Velarde C, Gotuzzo E, Cabrera J. Amoeba angeitic lesions of the central nervous system in Balamuthia mandrillaris amoebiasis. Hum Pathol 1999; 30: 269-73.

11.- Jayasekera S, Matin A, Sissons J, Maghsood A H, Khan N A. Balamuthia mandrillaris stimulates interleukin-6 release in primary human brain microvascular endothelial cells via a phosphatidyl inositol 3-kinase-dependent pathway. Microbes and
Infection 2005; 7: 134-47.

12.- Jayasekera S, Sissons J, Tucker J, Rogers C, Nolder D, Warhurst D, et al. Post-morten culture of Balamuthia mandrillaris from the brain and cerebrospinal fluid of a case of granulomatous amoebic meningoencephalitis. J Med Microbiol 2004; 53: 1007-12.

13.- Schuster F L, Dunnebacke T H, Booton G C, Yagi S, Kohlmeier CK, Glaser C, et al. Environmental isolation of Balamuthia mandrillaris associated with a case of amebic encephalitis. J Clin Microbiol 2003; 41: 3175-80.

14.- Yagi S, Booton G C, Visvesvara G S, Schuster F L. Detection of Balamuthia mitochondrial 16S rRNA gene DNA in clinical specimens by PCR. J Clin Microbiol 2005; 43: 3192-7.

15.- Deetz T R, Sawyer M H, Billman G, Schuster F L, Visvesvara G S. Successful treatment of Balamuthia amoebic encephalitis; presentation of 2 cases. Clin Infect Dis 2003; 37: 1304-12.

16.- Jung S, Schelper R L, Visvesvara G S, Chang $\mathrm{H}$ T. Balamuthia mandrillaris meningoencephalitis in an immunocompetent patient. An unusual case with a favorable outcome. Arch Pathol Lab Med 2004; 128: 466-8. 